\title{
Métodos de Remoção do Antibiótico Amoxicilina em Solução via Hidrólises Ácida e Alcalina
}

\author{
Taynara A. P. de Sá, Jerônimo R. O. Neto \& Thiago S. S. Ribeiro
}

\begin{abstract}
Os efluentes industriais gerados pelas indústrias podem causar sérios danos ao meio ambiente, se descartados indevidamente. Esses resíduos líquidos devem passar por processos de tratamento antes de serem liberados para os corpos receptores. As indústrias farmacêuticas geram efluentes com alta carga orgânica poluidora, destacando-se os antibióticos. O tratamento dos efluentes contendo antibióticos deve ser realizado de forma específica para que não prejudique os cursos de água e logo a fauna, a flora e a população de modo geral. Este trabalho teve como objetivo avaliar as melhores condições de hidrólise para promover a inativação do antibiótico amoxicilina em solução aquosa. Para esta avaliação foram realizados testes com variação de $\mathrm{pH}$ e tempo de exposição às condições estudadas, sendo analisados por cromatografia líquida de alta eficiência (HPLC/CLAE). Em relação ao tipo de hidrólise, verificou-se que a hidrólise alcalina se sobressai, sendo mais indicada. Os resultados obtidos sugerem que o método mais eficaz para o tratamento de efluentes industriais farmacêuticos contendo amoxicilina é a hidrólise alcalina em um pH de 12,5 e tempo de hidrólise de 60 minutos.
\end{abstract}

Palavras Chave: Efluentes Industriais; Antibióticos; Amoxicilina; Hidrólise; CLAE.

Industrial effluents generated by industries, if improperly disposed of, can cause serious damage to the environment. These liquid wastes must undergo treatment processes before they are released to the recipient bodies. Pharmaceutical industries generate effluents with high polluting organic load, especially antibiotics. The treatment of the effluents containing antibiotics must be carried out in a specific way so that it does not harm the water courses and soon the fauna, flora and the population. This work aimed to evaluate the best hydrolysis conditions to promote the inactivation of the antibiotic amoxicillin in aqueous solution. For this evaluation were made tests with $\mathrm{pH}$ variation and time of exposure to the studied conditions, being analyzed by high performance liquid chromatography (HPLC). In relation to the type of hydrolysis, whether acid or alkaline, it was verified that the alkaline hydrolysis protrudes, being more indicated. The results obtained suggest that the most effective method for the treatment of pharmaceutical industrial effluents containing amoxicillin is alkaline hydrolysis at $\mathrm{pH} 12.5$ and hydrolysis time of 60 minutes.

Keywords: Industrial Wastewater; Antibiotics; Amoxicillin; Hydrolysis; HPLC. 


\section{Introdução}

Um dos grandes problemas das indústrias é a geração de resíduos (sólidos, líquidos e gasosos) oriundos dos processos de produção, lavagens de equipamentos e sanitários de funcionários. Estes resíduos devem ser descartados de forma adequada para que não causem danos drásticos ao meio ambiente (ARAUJO, et al., 2008).

Os resíduos líquidos produzidos pelas indústrias são denominados efluentes industriais líquidos que possuem alta carga poluidora e se lançados aos corpos hídricos afetam suas características de forma negativa. Então faz-se necessário o tratamento prévio destes rejeitos utilizando-se processos, tanto físico-químicos quanto biológicos, variando de acordo com o tipo de poluente presente, para que este possa ser despejado de acordo com a determinação da legislação (FERREIRA, 2015).

Cada indústria aplica o tratamento conveniente com o efluente a ser tratado para que assim, possa se obter ao final do processo, um melhor resultado que não prejudique o meio ambiente em geral. A indústria farmacêutica gera efluentes com diversos tipos de poluentes, destacandose os antibióticos. Os despejos contendo antibióticos se lançados aos corpos receptores sem um tratamento prévio pode causar danos ao meio ambiente e logo à população (FAVARETTO et al., 2015).

A grande problemática dos antibióticos é que eles são recalcitrantes, possuindo biodegrabilidade muito baixa, o que ocasiona danos no meio ambiente, contaminando os recursos hídricos e também viabilizando o surgimento de microrganismos superresistentes (AQUINO, et al., 2013).

Dentre os métodos que podem ser utilizados na indústria farmacêutica para o controle de antibióticos estão os processos oxidativos avançados (POA) e as hidrólises ácida e alcalina (FRAU et al., 1996).

Diante ao exposto, este trabalho tem por objetivo analisar as melhores condições para a inativação do antibiótico amoxicilina via hidrólise, a fim de se propor uma aplicação, mais eficaz e viável, em Estações de Tratamento de Efluentes Industriais (ETEs) de indústrias farmacêuticas, visando melhorar também as condições de lançamentos de efluentes industriais nos corpos d'água.

\section{Metodologia}

As análises quantitativas das amostras de amoxicilina, após os testes de inativação, foram realizadas via cromatografia líquida de alta eficiência (CLAE), no laboratório do Núcleo de Estudos e Pesquisas TóxicoFarmacológicas (NEPET) da Faculdade de Farmácia da Universidade Federal de Goiás (UFG) em Goiânia - GO. Em todas as análises, utilizou-se um pHmetro da marca HANNA, modelo pH21, calibrado antes da realização de cada um dos testes.

Após a realização dos testes foram coletadas alíquotas de $1 \mathrm{~mL}$, colocado no vial e condicionado ao CLAE, da marca Shimadzu (modelo LC-20AT Prominence), com uma coluna C18 (150x4,6mm; $5 \mu \mathrm{m})$, fase móvel com Ácido fórmico $(0,2 \%)$ e Acetonitrila com proporção de 92:8, tempo de retenção de 3,5 minutos, tempo de corrida de 6 minutos, fluxo de $1 \mathrm{~mL} \mathrm{~min}^{-1}$, forno à $30^{\circ} \mathrm{C}$, comprimento de onda de $232 \mathrm{~nm}$, amostra de $10 \mathrm{mg} \mathrm{L}^{-1}$ e volume de $20 \mu \mathrm{L}$.

\section{TESTE DA VARIAÇÃO DO PH ALCALINO}

Foi preparada uma solução de amoxicilina com concentração igual a $10 \mathrm{mg} \mathrm{L}^{-1} \mathrm{e}$ verificado seu $\mathrm{pH}$ inicial que foi de 8,34. Após essa verificação, foram retiradas alíquotas da solução e colocadas em quatro béqueres, ajustando-se seus pHs como indicado na Figura $1 \mathrm{com}$ a adição de uma solução de hidróxido de sódio 0,5 mol.L-1 .

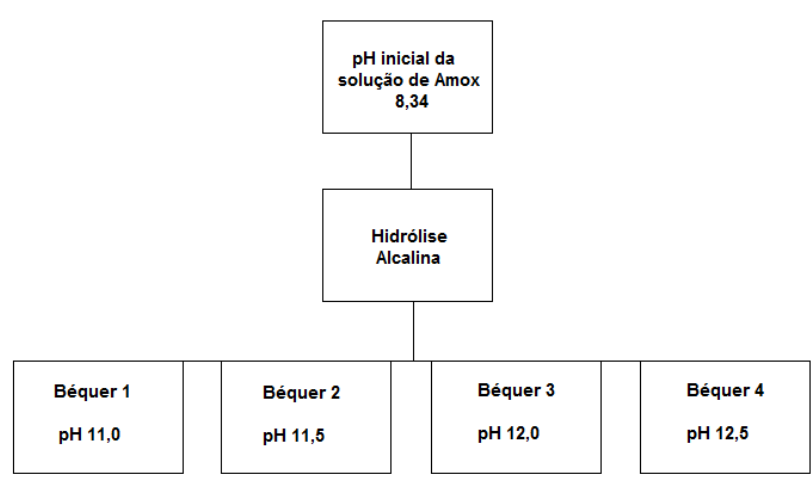

Figura 1. Fluxograma da variação dos pHs alcalinos. 


\section{TESTE DE VARIAÇÃO DO PH ÁCIDO}

Semelhantemente, foram retiradas alíquotas da solução de amoxicilina com $\mathrm{pH}$ inicial igual à 8,34 , colocadas em dois béqueres e ajustadas aos seguintes pHs ácidos mostrados pela Figura 2 com a adição de uma solução de ácido clorídrico $0,5 \mathrm{~mol} \cdot \mathrm{L}^{-1}$.

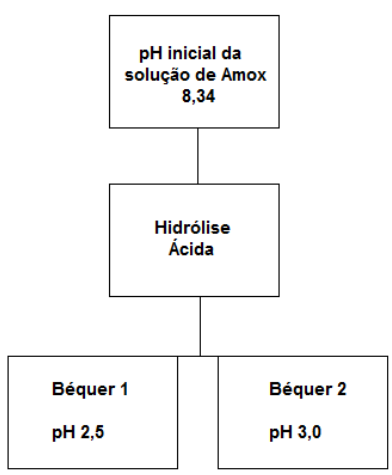

Figura 2. Fluxograma da variação de $\mathrm{pH}$ ácido.

\section{TESTE DO TEMPO DE HIDRÓLISE}

Logo após os ajustes dos pH's para a realização dos testes de hidrólises, verificou-se o tempo ideal de exposição para a inativação da amoxicilina, através da coleta de amostras em intervalos de 1, 5, 10, 15, 20, 30 minutos, conforme mostram as Figuras 3 e 4.

Para os testes em pH's alcalinos, as soluções foram neutralizadas antes de serem condicionadas ao CLAE, pois a coluna utilizada limita seu uso entre o $\mathrm{pH} 2,0$ a 10,0.
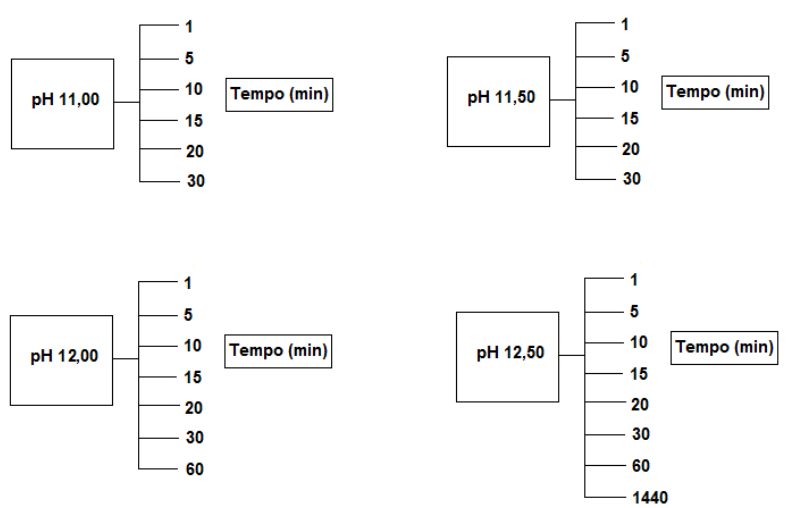

Figura 3. Fluxograma $\mathrm{pHs}$ alcalinos e seus determinados tempos de exposição a hidrólise básica.
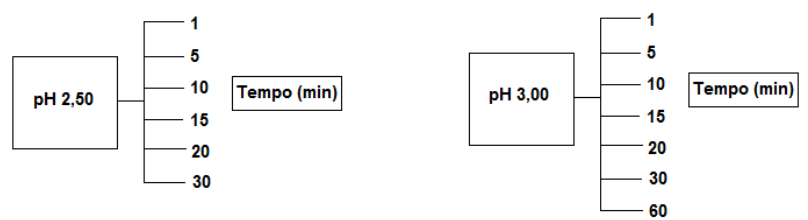

Figura 4: Fluxograma dos pHs ácidos e seus determinados tempos de exposição a hidrólise ácida.

\section{CURVA DE CALIBRAÇÃO}

Foram preparadas soluções de Amoxicilina nas seguintes concentrações para a confecção do gráfico da curva de calibração: $0,1 \mathrm{mg} \mathrm{L}^{-1} ; 0,5 \mathrm{mg} \mathrm{L}^{-1} ; 1,0 \mathrm{mg} \mathrm{L}^{-1} ; 2,5 \mathrm{mg} \mathrm{L}^{-1} ; 5$ $\mathrm{mg} \mathrm{L}{ }^{-1} ; 10 \mathrm{mg} \mathrm{L}^{-1} ; 25 \mathrm{mg} \mathrm{L}^{-1} ; 50 \mathrm{mg} \mathrm{L}^{-1} ; 100 \mathrm{mg} \mathrm{L}^{-1}$.

\section{Resultados e Discussão}

Os resultados obtidos foram quantificados através do equipamento Cromatografia Líquido de Alta Eficiência (CLAE), situado no Laboratório da Faculdade de Farmácia da UFG em Goiânia-GO.

\section{TESTE DE VARIAÇÃO DO PH ALCALINO}

A hidrólise alcalina do anel $\beta$-lactâmico foi feita a partir da exposição das alíquotas retiradas da solução de Amoxicilina (Amox) em pH alcalino, onde cada béquer com a solução foi submetido ao $\mathrm{pH}$ demonstrado na metodologia, através da adição de uma solução de hidróxido de sódio $0,5 \mathrm{~mol} \mathrm{~L}^{-1}$.

\section{TESTE DE VARIAÇÃO DO PH ÁCIDO}

Com o pH inicial da solução de Amox sendo 8,34, cada alíquota retirada foi condicionada para a realização da hidrólise ácida com os pHs 2,5 e 3 através da adição de uma solução de ácido clorídrico $0,5 \mathrm{~mol} \mathrm{~L}^{-1}$.

\section{TESTE DO TEMPO DE HIDRÓLISE}

O teste do tempo de hidrólise foi realizado determinando o melhor tempo de exposição à hidrólise ácida e alcalina para cada faixa de $\mathrm{pH}$. A partir da medida de intensidade do pico dado nos gráficos de quantificação em CLAE, conseguiu-se analisar o melhor tempo de hidrólise com maior inativação da amostra de Amox. 
Nos gráficos mostrados pelas figuras a seguir o pico de intensidade observado em torno de 4 minutos de corrida é referente à molécula de Amoxicilina, e supõe-se que o pico de intensidade em torno de 3,5 minutos seja um intermediário da hidrólise do anel $\beta$-lactâmico e que pico por volta de 3 minutos seja a formação do produto de inativação, ou seja, o anel $\beta$-lactâmico já hidrolisado.

O gráfico da Figura 5 mostra os resultados da exposição da solução de Amox ao pH 11.

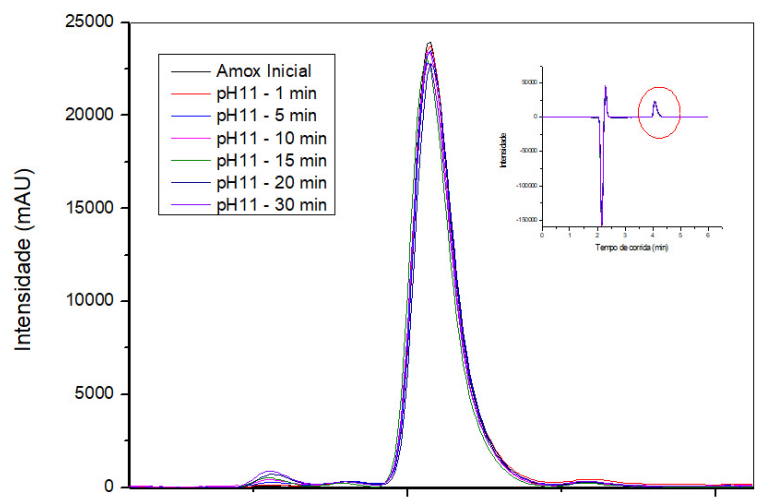

Figura 5. Gráfico da inativação da Amoxicilina em solução no pH 11.

Fonte: Autor. Análise realizada em cromatógrafo Shimadzu,

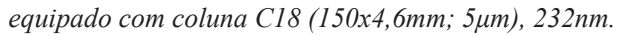

Observou-se que no pH 11 até 30 minutos, não há uma inativação significativa da molécula de Amox, pois o pico em 4 minutos de corrida continua com alta intensidade. Verificou-se também que a maior inativação ocorre em 30 minutos, observando a formação do possível intermediário do processo de hidrólise em torno em tempo de 5 minutos de hidrólise.

Os resultados dos testes com $\mathrm{pH}$ 11,5 estão mostrados na Figura 6. Notou-se uma pequena inativação da amostra de Amox em 30 minutos, porém não suficiente para a remoção total do antibiótico em solução, pois o pico de intensidade continua alto mesmo após esse tempo de análise. No gráfico observou-se o surgimento em 5 minutos de hidrólise do possível intermediário da reação.

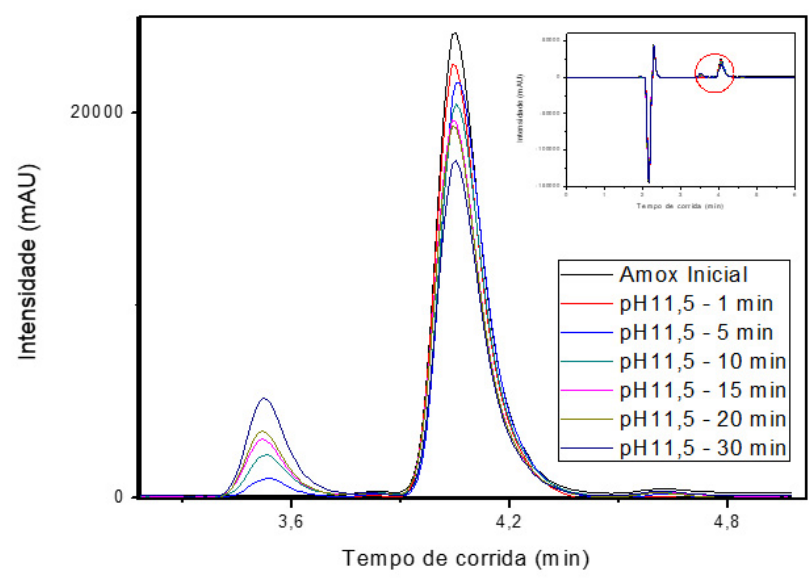

Figura 6. Gráfico da inativação da Amoxicilina em solução no pH 11,5.

As análises da hidrólise alcalina em pH 12 estão demonstradas no gráfico da Figura 7, onde foi possível observar a redução do antibiótico Amoxicilina já em 1 minuto e em 60 minutos tem-se o melhor tempo da inativação da molécula, porém o pico de intensidade continua suficientemente alto indicando que não houve inativação total da molécula. Notou-se o surgimento do suposto intermediário da reação de hidrólise já em 1 minuto de hidrólise e a formação do possível produto de inativação em 10 minutos de hidrólise.

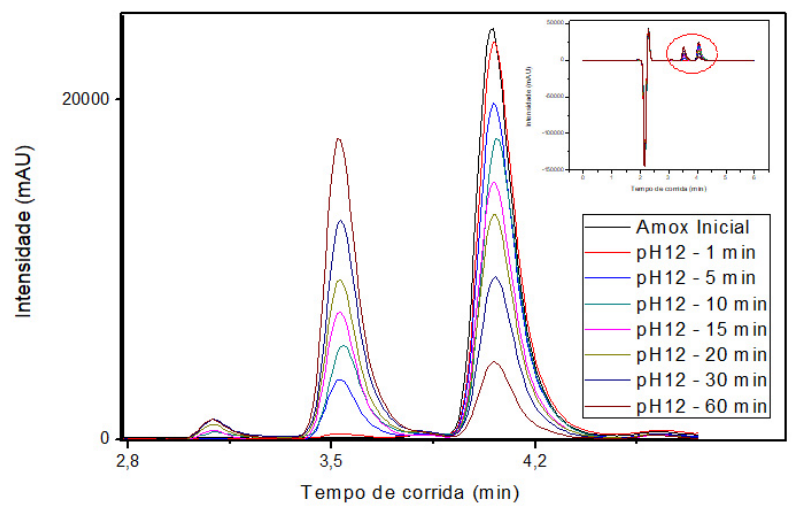

Figura 7. Gráfico da inativação da Amoxicilina em solução no pH 12. Fonte: Autor. Análise realizada em cromatógrafo Shimadzu, equipado com coluna C18 (150x4,6mm; $5 \mu \mathrm{m}), 232 \mathrm{~nm}$. 
Na Figura 8 tem-se o gráfico da inativação da Amox em pH alcalino de 12,5. Foi possível verificar a completa redução do pico de intensidade em 60 minutos de hidrólise que aponta a completa inativação da Amoxicilina. Verificou-se também a formação do hipotético intermediário de inativação sendo formado em 1 minuto de hidrólise e em 10 minutos de hidrólise observou-se a formação do suposto produto de inativação da molécula.

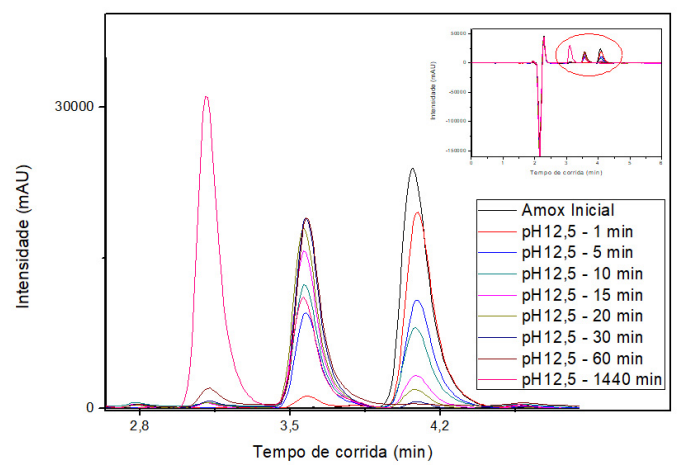

Figura 8. Gráfico da inativação da Amoxicilina em solução no pH 12,5.

Fonte: Autor. Análise realizada em cromatógrafo Shimadzu, equipado

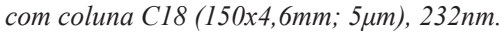

$\mathrm{Na}$ hidrólise ácida em $\mathrm{pH}$ de 2,5 tem-se o gráfico representado na Figura 9, em que é possível notar uma pequena redução do pico de intensidade da amostra de Amox em 30 minutos de hidrólise. Essa redução não foi significativa para a inativação do antibiótico, pois o pico de intensidade ainda se apresenta muito alto após os tempos de análises.

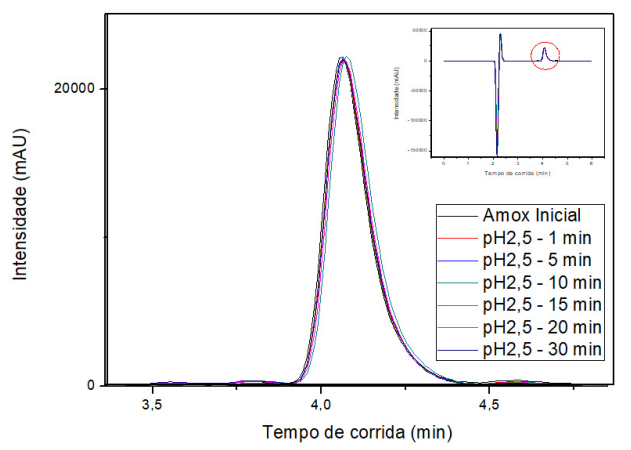

Figura 9. Gráfico da inativação da Amoxicilina em solução no pH 2,5. Fonte: Autor. Análise realizada em cromatógrafo Shimadzu, equipado com coluna C18 (150x4,6mm; 5 $\mathrm{m}$ ), 232nm.
A Figura 10 demonstra o gráfico da inativação da molécula de Amox em pH ácido 3 onde é possível observar uma pequena inativação da Amoxicilina em 60 minutos, porém essa inativação não é considerável para a remoção total da molécula, pois o pico de intensidade ainda se apresenta muito alto.

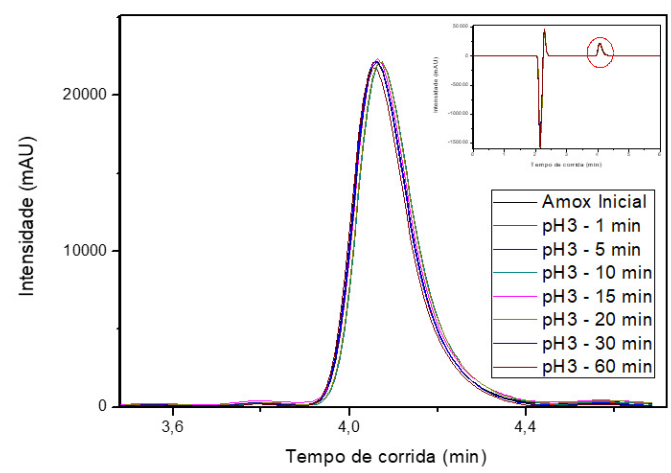

Figura 10. Gráfico da inativação da Amoxicilina em solução no pH 3. Fonte: Autor. Análise realizada em cromatógrafo Shimadzu, equipado com coluna C18 (150x4,6mm; 5 $\mu \mathrm{m}), 232 \mathrm{~nm}$.

Com o intuito de se realizar um comparativo entre todas as situações analisadas, é que a Figura 11 mostra a relação dos melhores tempos de hidrólises de cada $\mathrm{pH}$.

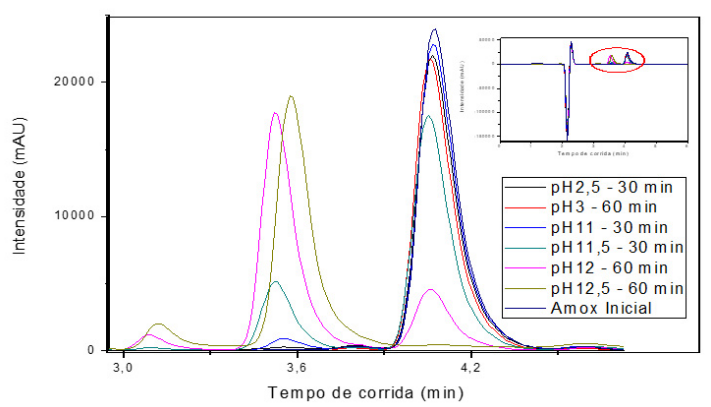

Figura 11. Gráfico com os melhores tempos de inativação da Amoxicilina em solução de cada $\mathrm{pH}$.

Fonte: Autor. Análise realizada em cromatógrafo Shimadzu, equipado com coluna C18 (150x4,6mm; $5 \mu \mathrm{m}), 232 \mathrm{~nm}$. 
Através do gráfico foi possível avaliar a eficácia de cada $\mathrm{pH}$ em seus melhores tempos de hidrólise. Os pHs 2,5 e 3 da hidrólise ácida e $\mathrm{pH} 11$ da hidrólise alcalina foram os menos efetivos na inativação da Amoxicilina, visto que houve pouca redução do pico em 4,0 minutos de corrida, atribuído à molécula de Amox. Com isso verificou-se inapropriada a utilização do método de hidrólise ácida e alcalina com $\mathrm{pH}$ 11 em Estações de Tratamento de Efluentes de indústrias farmacêuticas por ter baixa eficiência na remoção da Amoxicilina.

Já nos testes que utilizaram os pHs 11,5 e 12 notou-se a redução do pico em 4,0 minutos de corrida, atribuindo-se à inativação do antibiótico em solução, nos melhores tempos de hidrólises, porém não de forma total, o que indica que seria inviável a aplicação deste método nesse pHs em indústrias contendo Amoxicilina em seus efluentes, pois necessitaria de um tempo maior de hidrólise na inativação total da molécula de Amox.

$\mathrm{Na}$ hidrólise alcalina com pH 12,5 em 60 minutos de testes ocorreu a inativação total da molécula de Amox observado através da redução completa do pico em 4,0 minutos de corrida, atribuído à molécula de Amoxicilina. Diante disso verificou-se que a hidrólise alcalina com $\mathrm{pH}$ 12,5 em 60 minutos seriam as condições mais apropriadas para a aplicação em indústrias farmacêuticas.

Para a determinação das concentrações finais da Amox em solução após os testes de inativação, foi confeccionado um gráfico da curva de calibração, mostrado na Figura 12.

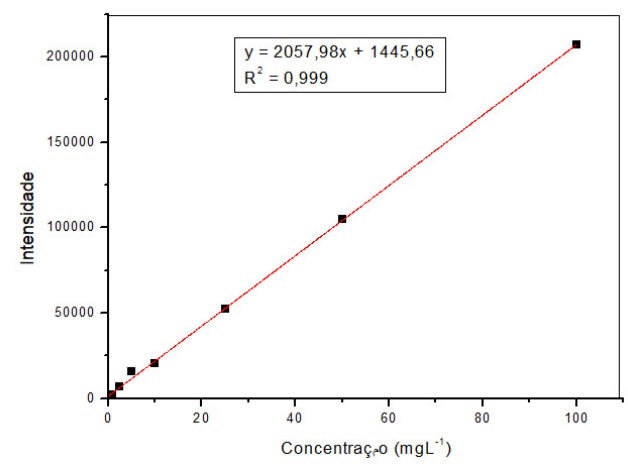

Figura 12. Gráfico da curva de calibração.
Com a equação da reta obtida a partir de curva de calibração, com concentrações de Amoxicilina de $0,1 \mathrm{mg} \mathrm{L}^{-1}$ a $100 \mathrm{mg} \mathrm{L}^{-1}$, foi possível calcular a concentração do antibiótico após os testes de hidrólises em seus melhores tempos e as porcentagens de remoção, mostrados na Tabela 1 .

Tabela 1. Relação da concentração da Amoxicilina antes e depois das hidrólises em seus melhores tempos.

\begin{tabular}{|c|c|c|}
\hline $\begin{array}{c}\text { pH e melhor } \\
\text { Tempo de Hidrólise }\end{array}$ & $\begin{array}{c}\text { Concentração } \\
\left.\text { (mgL }^{-1}\right)\end{array}$ & Remoção \\
\hline Amox Inicial & 10,9 & - \\
\hline $\mathrm{pH} 11-30 \mathrm{~min}$ & 10,3 & $5,5 \%$ \\
\hline $\mathrm{pH} 11,5-30 \mathrm{~min}$ & 7,1 & $34,5 \%$ \\
\hline $\mathrm{pH} 12-60 \mathrm{~min}$ & 1,4 & $87,0 \%$ \\
\hline $\mathrm{pH} 12,5-60 \mathrm{~min}$ & 0,0 & $100,0 \%$ \\
\hline $\mathrm{pH} 2,5-30 \mathrm{~min}$ & 9,8 & $10,0 \%$ \\
\hline $\mathrm{pH} 3-60 \mathrm{~min}$ & 9,4 & $14,1 \%$ \\
\hline
\end{tabular}

A Figura 13 mostra a porcentagem de remoção da Amoxicilina em cada $\mathrm{pH}$.

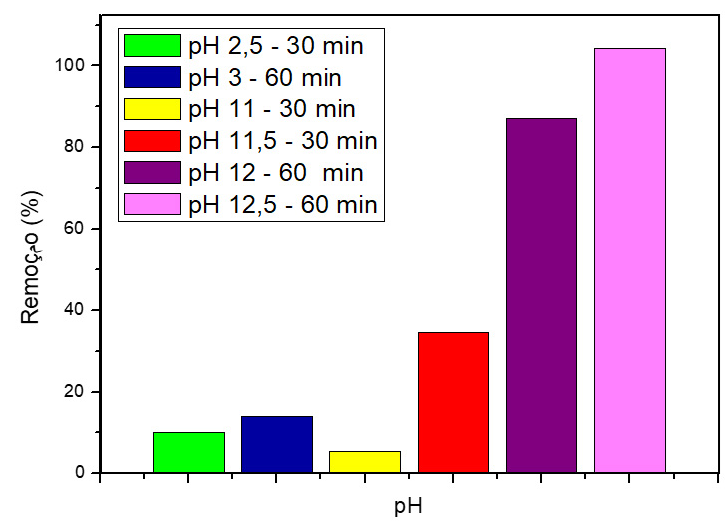

Figura 13. Gráfico em barras da relação dos pHs e suas porcentagens de remoção da molécula de amoxicilina.

Notou-se novamente que os pHs 2,5, 3 e 11 não foram eficazes na inativação da molécula de Amox com porcentagem de remoção de $10 \%, 14,1 \%$ e 5,5\% respectivamente, sendo muito baixas quando comparadas aos valores das outras condições estudadas. 
No pH 11,5 houve remoção de 34,5\% de Amox. Em pH 12 a remoção da foi de 87\%, que é bastante eficiente, porém necessitaria de um maior tempo para a total remoção do antibiótico estudado, o que inviabilizaria a utilização em uma indústria que utiliza processos contínuos de tratamentos de efluentes. Já no pH 12,5 houve a remoção de 100\% da molécula de amoxicilina em 60 minutos de exposição, tornando então este método mais eficaz e apropriado para a aplicação em futuras estações de tratamento de efluentes industriais.

Em relação à hidrólise ácida, além dos baixos valores de inativação de amox em solução, deve-se levar em consideração que a utilização de pHs baixos em altas concentrações pode ocasionar a corrosão de tubulações e tanques metálicos da estação.

Já o uso da hidrólise alcalina é viável em ETE's de indústrias contendo amoxicilina visto que não danifica os equipamentos do processo. Além disso, o método é eficiente em pH 12,5 e tempo de hidrólise de 60 minutos, observado nestas condições o menor tempo de exposição à hidrólise e menor gasto de soluções alcalinas, gerando um menor custo. A utilização de pHs inferiores à 12,5 acarreta um maior tempo de exposição e pHs acima de 12,5 necessitam de uso de soluções alcalinas mais concentradas ou em maior quantidade o que leva à um gasto financeiro maior, sendo inviável para uma indústria que busca sempre custo benefício mais adequado.

\section{Conclusões}

Foi realizada a avaliação do melhor método para a inativação de amoxicilina via hidrólise ácida e alcalina e verificação do melhor $\mathrm{pH}$ e tempo de hidrólise para a total inativação do antibiótico estudado.

A hidrólise ácida analisada nos pHs 2,5 e 3 não foi efetiva visto que não foi alcançada a inativação total da molécula de amoxicilina mesmo em tempos altos de hidrólise, então sua utilização seria inviável em indústrias tanto pela pouca eficiência do método quanto pela possível danificação que pHs ácidos ocasionam nos equipamentos metálicos dos processos.

A hidrólise alcalina com pH 12,5 e tempo de 60 minutos foi a técnica mais eficiente visto que utiliza menor tempo para a total remoção da amoxicilina, observando $100 \%$ de inativação. O estudo de metodologias de remoção de antibióticos em efluentes industriais é de grande relevância para proporcionar às indústrias farmacêuticas o método mais eficaz e viável evitando desperdícios econômicos e de tempo.

Sugere-se para trabalhos futuros a determinação dos intermediários do processo de hidrólise, verificando-se também suas ações antibióticas e suas possíveis toxicidades.

\section{Agradecimentos}

Ao Laboratório de Análise Instrumental da UEG, CCET. Ao Laboratório NEPET-UFG.

\section{Referências Bibliográficas}

1. Almeida, E., Assalin, M.R., Rosa, M.A., Dúran, N. Wastewater treatment by oxidation with ozone. Tratamento de efluentes industriais por processos oxidativos na presença de ozônio. Química Nova, v.27, n5, p.818-824, 2004.

2. Aquino, S.F.; Brandt, E.M.F.; Chernicharo, C.A.L. Remoção de fármacos e desreguladores endócrinos em estações de tratamento de esgoto: revisão da literatura. Engenharia Sanitária Ambiental, v. 18, n. 3, p. 187-204, 2013.

3. Araújo, B.C.S.; Silva, J.M.B.; Wada, R.; Filho, S.R.O. Tratamento de efluentes industriais. Revista Ciências do Ambiente On-line, v.04, $\mathrm{n}^{\circ} 02,2008$.

4. Baptistucci, C.B. Inativação do antibiótico ciprofloxacina em solução aquosa por meio de processo oxidativo avançado baseado em ozônio. Dissertação de mestrado, Escola Politécnica da Universidade de São Paulo. São Paulo, 2012.

5. Brasil, Associação Brasileira De Normas Técnicas. NBR-9800: Critérios para Lançamento de Efluentes Líquidos Industriais no Sistema Coletor Público de Esgoto Sanitário. Rio de Janeiro, 1987.

6. Brasil, Resolução Conama $N^{0} 357$, Conselho Nacional do Meio Ambiente, 17 de março de $\mathbf{2 0 0 5}$.

7. Brasil, Resolução Conama $\mathrm{N}^{\circ} 430$, Conselho Nacional do Meio Ambiente, 13 de maio de 2011.

8. Deshpande, A.D.; Baheti, K.G.; Chatterjee, N.R. Degradation of $\beta$-lactam antibiotics. Current Science, v.87, nº12, 2004.

9. Dezotti, M. Processos e técnicas para o controle ambiental de 
efluentes líquidos. Série Escola Piloto de Engenharia Química COPPE/UFRJ, v.05, p. 360, Editora E-papers, 2008.

10. Favaretto, D.P.C.; Brião, V.B.; Colla, L.M.; Hemkemeier, M. Análise técnica do processo de tratamento de efluentes de empresa de laticínios da região de Passo Fundo/RS. Revista CIATEC-UPF, v. 7, n. 2, p. 18-30, 2015.

11. Ferreira, E.F. Análise dos Processos e Grau de Tratamento dos Efluentes Industriais: No segmento de laticínios e tabacos. e-RAC, v.05, n. 1, 2015.

12. Ferreira, J.A.M. Tratamento de efluentes.. VIII Encontro Nacional Sobre Métodos dos Laboratórios da Embrapa. Jaguariúna -SP, 2003. Disponível em < http://www.cnpsa.embrapa.br/met/images/ arquivos/08MET/Palestras/tratamentoefluentes.pdf $>$ página acessada em 05 de julho de $\mathbf{2 0 1 5}$

13. Frau, J.; Coll, M.; Donoso, J.; Muñoz, F.; Vilanova, B.; Garcia-Blanco, F. Alkaline and acidic hydrolysis of the $\beta$-lactam ring. Eletronic Journal Of Theoretical Chemistry, v. 2, p. 56-65 (1997). 1996.

14. Nogueira, R.; Trovó, A.; Aguera, A.; Fernandez-Alba, A.; Malato, S. Degradation of the antibiotic amoxicillin by photo-fenton process - Chemical and toxicological assessment. Water Research, $n^{\circ} 45$, p.1394-1402, 2011.

15. Pereira, R.S. Identificação e caracterização das fontes de poluição em sistemas hídricos. Revista Eletrônica de Recursos Hídricos. IPH - UFRGS. v.1, n.1, p. 20-36, 2004. Disponível em < http: //www. abrh.org.brinformacoesrerh.pdf $>$ página acessada em 30 de junho de 2015.

16. Rocha J.S.; Vargem, D.S.; Santos, W.; Ferreira, G.F.; Vieira, E.F.; Menezes, E.D. Inativação por hidrólise alcalina de antibióticos $\beta$-lactâmicos, cefalosporínicos e penicilâmicos. Ensaios e Ciência: Ciências Biológicas, Agrárias e da Saúde, v. 17, n. 1, p. 19-31. Universidade Anhanguera, Campo Grande, 2013.

17. Silva, F.K.; Eyng, J. O tratamento de águas residuais de indústria de laticínios: Um estudo comparativo entre os métodos de tratamento com biofiltro e com o sistema convencional de lagoas. Revista gest. Sust. Ambiental, v.01, nº2, p.4-22. Florianópolis, 2013.

18. Vasconcelos, N.F.; Pinheiro, J.A.; Brígida, A.I.S.; Filho, M.S.M.S.; Rosa, M.F. Otimização do processo de obtenção de nanocelulose do pseudocaule da bananeira por hidrólise ácida. Embrapa Instrumentação, Universidade Federal do Ceará. São Carlos - SP, 2013.

19. Vasconcelos, O.M.S.R. Inativação do antibiótico amoxicilina em efluente de indústria farmacêutica. Dissertação de pós-graduação, Universidade Federal de Minas Gerais. Belo Horizonte - MG, 2011.

20. Von Sperling, M. Princípio de tratamento de águas residuárias. Introdução à qualidade das águas e ao tratamento de esgotos. Editora UFMG. Departamento de Engenharia Sanitária e Ambiental DESA. Universidade Federal de Minas Gerais - MG, 2005.

\section{Taynara A. P. de Sá', Jerônimo

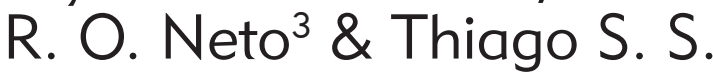 Ribeiro ${ }^{1,2}$}

${ }^{1}$ Universidade Estadual de Goiás, Campus Anápolis de Ciências Exatas e Tecnológicas. BR-153, 3105 - Fazenda Barreiro do Meio, Anápolis - GO, 75132-903.

${ }^{2}$ Faculdade Metropolitana de Anápolis - FAMA. Av. Fernando Costa, 49 Vila Jaiara St. Norte, Anápolis - GO, 75064-780

${ }^{3}$ NEPET - Núcleo de Estudos e Pesquisas Tóxico-Farmacológicas, Faculdade de Farmácia, Universidade Federal de Goiás. Rua 240, esquina

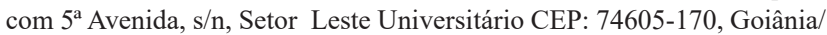
Goiás - Brasil.

*E-mail: thiago cunha@hotmail.com 